\title{
Efecto de la edad y la localidad en la concentración de cadmio y cobre en el hígado de la gaviota dominicana Larus dominicanus
}

Effect of age and location in the concentration of cadmium and copper in the liver of the Kelp gull Larus dominicanus

\author{
Maritza Cortés ${ }^{1}$ y Guillermo Luna-Jorquera ${ }^{1,2}$
}

\author{
${ }^{1}$ Laboratorio de Ecología y Diversidad de Aves Marinas, Departamento de Biología Marina, Facultad de Ciencias del Mar, \\ Universidad Católica del Norte, Larrondo 1281, Coquimbo, Chile \\ ${ }^{2}$ Centro de Estudios Avanzados en Zonas Áridas (CEAZA), Larrondo 1281, Coquimbo, Chile. gluna@ucn.cl
}

\begin{abstract}
We analyzed $\mathrm{Cu}$ and $\mathrm{Cd}$ liver concentration in adults and fledglings of Kelp gull from an urban (Coquimbo city) and a wild (Pájaros Islet) colony. Cu concentration was $15.57 \pm 0.079 \mu \mathrm{g} \mathrm{g}^{-1}$ dry weight for adults and fledglings of both colonies. Adults $\mathrm{Cd}$ concentration from both colonies was $9.72 \pm 0.110 \mu \mathrm{g} \mathrm{g}^{-1} \mathrm{dw}$, being $194 \%$ of the level considered toxic but in the range for seabirds. In the urban fledglings $\mathrm{Cd}$ was below the detection level. Wild fledgling showed higher concentration of $1.54 \pm 0.064 \mu \mathrm{g} \mathrm{g}^{-1} \mathrm{dw}$ which probably reflects a biomagnification process, because parents kleptoparasite and consume eggs and chicks of other seabirds.
\end{abstract}

Key words: Heavy metals, seabirds, Kelp gull, biomagnification

\section{INTRODUCCIÓN}

Las actividades humanas que se desarrollan en las costas de Chile a menudo generan impactos negativos que afectan el paisaje, la salud y a veces la muerte de organismos silvestres (Bravo et al. 2009, Thiel et al. 2011). A pesar que los metales pesados se encuentran en forma natural en el ambiente marino, se reconoce que las actividades industriales que descargan sus desechos al mar han provocado un aumento de algunas sustancias en concentraciones peligrosas para la salud de los ecosistemas (Moriarty 1983, Zúñiga 1998, ICES 1999). Entre los metales pesados el mercurio (Hg), el plomo $(\mathrm{Pb})$, el cadmio (Cd) y el cobre $(\mathrm{Cu})$, se consideran como los más dañinos para organismos y ambientes naturales (Zúñiga 1998).

La presencia de metales y sustancias contaminantes en aves marinas de Chile ha sido investigada a varios niveles de escalas espaciales. A nivel local, en playas contaminadas con relaves mineros, se determinaron altos niveles de cadmio y cobre en un grupo de 6 especies (Vermeer \& Castilla 1991). A una escala espacial mayor comprendida entre Algarrobo (Región de Valparaíso, Chile central) y la Isla Magdalena (Estrecho de Magallanes, Chile austral), la concentración de compuestos organoclorados en huevos de gaviota dominicana han mostrado un patrón de variación norte-sur en el cual las mayores concentraciones se encontraron en el norte (Muñoz \& Becker 1999). A una escala espacial aún mayor, desde el Parque Nacional Pan de Azúcar (Región de Atacama, norte de Chile) hasta Puerto Williams (Región de Magallanes) y el Archipiélago de Juan Fernández, la concentración de mercurio en 22 especies de aves marinas fue más baja que los niveles considerados letales y fue influenciada por la ubicación geográfica, los hábitos alimenticios y la filogenia (Ochoa-Acuña et al. 2002). Estos y otros estudios (e.g., Muñoz et al. 2003) aportan información de línea base, que para el caso de aves marinas con distribución amplia permite establecer comparaciones entre distintos puntos a lo largo de la costa. Sin embargo, estos trabajos no consideran la variación intraespecífica en la concentración de contaminantes a escalas espaciales pequeñas (i.e., $<100$ $\mathrm{km}$ ) que consideren la edad de los individuos y la existencia de otras colonias cercanas. Desde el comienzo de la temporada reproductiva y hasta el final del periodo de crianza, las aves marinas generalmente se alimentan en zonas cercanas a la colonia (Shealer 2002). Por lo tanto, la concentración de contaminantes en los juveniles usualmente se relaciona con los niveles existentes en el área definida por el rango de hogar y los hábitos de forrajeo de los padres durante la crianza (ICES 1999). 
En Chile no existe información para aves marinas que relacione los niveles de metales pesados entre individuos de diferentes edades y distintas colonias reproductivas, urbanas y silvestres, a una escala espacial pequeña, que permita determinar variaciones locales y contaminantes de interés con el objeto de implementar programas de monitoreo. En el Sistema Costero de Coquimbo (SCC), la gaviota dominicana nidifica en colonias silvestres de hasta 2000 parejas ubicadas principalmente en la isla Pájaros 1 (Simeone et al. 2003), sin embargo, existen colonias urbanas ubicadas en los edificios de la ciudad. Se estima que la cantidad de nidos en algunos edificios puede llegar a ca. 1 nido cada 2 metros cuadrados (Kozertka 2002). La gaviota dominicana es una especie común en el hemisferio sur (Harrison 1983), no presenta problemas de conservación (Birdlife 2011) y se le considera un buen bioindicador de las concentraciones de contaminantes en el ambiente marino costero (e.g., Muñoz et al. 2003). En el SCC la gaviota dominicana es un depredador tope de hábitos oportunista y generalista que presenta una fuerte dependencia de las fuentes de alimentación de origen antrópico (i.e., basuras y descartes de la pesquería) (Weichler et al. 2004, Villablanca et al. 2007). La dieta de los adultos cambia de acuerdo a la fase del estado reproductivo en que se encuentren (Ludynia et al. 2005). De este modo, para la gaviota dominicana en el SCC, es posible esperar que, a) los pollos de colonias urbanas y silvestres presenten niveles de metales pesados más bajos que los adultos, debido a que han estado expuestos por menos tiempo a las fuentes naturales y antrópicas de los contaminantes, y b) los pollos deberían presentar diferencias entre colonias debido a diferencias en la dieta de los padres condicionada por la oferta a nivel local. En el caso de los adultos no se deberían encontrar diferencias significativas en los contaminantes por efecto de la colonia en que nidifican, ya que una vez terminado el periodo reproductivo se dispersan ampliamente. Consecuentemente, el objetivo de este estudio fue analizar la concentración de Cd y Cu hepático en adultos y pollos de gaviota dominicana de una colonia silvestre (Isla Pájaros) y una urbana (Bahía La Herradura), ambas ubicadas en el SCC.

\section{MATERIALES Y MÉTODOS}

Durante el periodo reproductivo de enero de 2002, se capturaron pollos y adultos de gaviota dominicana en una colonia urbana ubicada en la Bahía La Herradura $\left(30^{\circ} 01^{\prime} \mathrm{S}\right)$ y en una colonia silvestre ubicada en Isla Pájaros (2932’S). Estas colonias están separadas por ca.
$47 \mathrm{~km}$. Los pollos volantones (edad ca. 40-42 días) se capturaron directamente en el nido y los adultos reproductivos (edad $>4$ años) fueron cazados con honda elástica cerca de sus nidos (Autorización Exenta № 3201, DIPROREN, SAG). En cada colonia se capturaron cinco individuos por grupo de edad. Cada individuo (adulto o juvenil) provino de nidos diferentes, sumando un total de 20 animales. Las aves fueron sacrificadas con cloroformo en una cámara cerrada. Previo a la disección se registró el peso corporal de cada animal con una balanza semi-analítica (exactitud $0,1 \mathrm{~g}$ ). Las aves fueron disectadas utilizando instrumental desechable de plástico para extraer el hígado, el que fue pesado y almacenado en bolsas plásticas herméticas (Ziploc $\left.{ }^{\circledR}\right)$ a $-5^{\circ} \mathrm{C}$. Cada hígado fue examinado visualmente para detectar la presencia o ausencia de necrosis.

Para determinar la concentración de Cd y Cu aplicamos el protocolo sugerido por Stegen et al. (2000). Cada hígado se desmenuzó y se llevó una temperatura de $-20^{\circ} \mathrm{C}$, para luego secar en un liofilizador (Leybold Mod. Lyavac GT.2) en rangos de temperatura de $8-15^{\circ} \mathrm{C}$ a presión constante de 0,01 milibar por $c a$. $6 \mathrm{hr}$. Una vez seco, cada hígado fue homogenizado en un molino de zirconio $\left(\mathrm{ZrO}_{2}\right.$, Fritsh, Idar-Oberstein, Germany, Planetary Mill Pullverisette) a $225 \mathrm{rpm}$ por $60 \mathrm{~min}$. De cada homogenizado se tomó una muestra de 0,2 g para su digestión con $2 \mathrm{~mL}$ de ácido nítrico (s.p. Merck) en crisoles de cuarzo (suprasil de 50 $\mathrm{mL}$ ) cubiertos con un vidrio reloj de cuarzo. Los recipientes fueron colocados a temperatura ambiente, hasta que el material fue disuelto. Luego, los recipientes se sometieron a una temperatura de $20^{\circ} \mathrm{C}$ la cual fue aumentando gradualmente hasta los $330^{\circ} \mathrm{C}$. Siguiendo este procedimiento, se realizaron dos digestiones adicionales por cada muestra de hígado y se preparó un estándar de referencia y un blanco para determinar la precisión del método. El estándar consistió en el material de referencia ‘hígado de bovino’ NBS 1577a (s.p. Merck). Las diferencias máximas observadas entre los valores de referencia y los valores encontrados en las muestras fueron de $5 \%$ y 4,2\% para el $\mathrm{Cd}$ y el $\mathrm{Cu}$, respectivamente.

El resultado de la digestión fue diluido con agua desionizada obtenida de Milli-Q-Water-PurificationSystem (Millipore, Germany). La solución resultante fue transferida a frascos de polietileno de $10 \mathrm{~mL}$ y se llevó a aforo con agua desionizada. Las concentraciones de $\mathrm{Cd}$ y Cu en las muestras, así como en los estándares y blancos, fueron medidas en un espectrofotómetro de absorción atómica Perkin Elmer Analyst 700 cuyo límite de detección fue de $0,01 \mu \mathrm{g} \mathrm{g}^{-1}$. Todos los datos se presentan en 
$\mu \mathrm{g} \mathrm{g}{ }^{-1}$ de peso seco (ps). Con fines comparativos los valores en peso húmedo reportados en la literatura, fueron aproximados a peso seco usando un factor de conversión de 0,38 calculado a partir de los datos en Mahoney \& Jehl (1984) para aves marinas.

Debido a que ambos metales estudiados proceden de los mismos individuos, se aplicó un test multivariado de análisis de varianza (MANOVA, Scheiner 1993, Quinn \& Keough 2002), de dos factores. El primer factor Colonia, incluye a las colonias de Isla Pájaros y La Herradura, y el segundo factor Edad, incluye a los individuos Adultos y Volantones. Ambas variables respuestas (Cd y Cu) cumplieron con los requisitos de normalidad marginal (test de Shapiro-Wilks, $P>0,41$ ), normalidad multivariada (prueba de Henze-Zirkler $=0,349 ; P=0,827$ ) $\mathrm{y}$ homocedasticidad de la varianza (prueba de Levene $P>>$ $0,05)$. Todos estos supuestos se cumplieron después de transformar los datos mediante $\log _{10}(X i+1)$. Las pruebas estadísticas fueron trabajadas con $\alpha=0,05$. Para informar y discutir los resultados se reportan la media y el error estándar, en su escala original.

\section{RESULTADOS Y DISCUSIÓN}

El examen visual practicado a los hígados de los adultos y pollos de gaviota dominicana, reveló que ninguno de ellos presentaba necrosis. En aves, la necrosis hepática está relacionada con la exposición prolongada a altas concentraciones de metales pesados (e.g., Vermeer \& Castilla 1991, Burger \& Gochfeld 2002). Aunque este examen sólo puede detectar daños a niveles macroscópicos, es útil como indicador del estado de salud general de las aves (Burger \& Gochfeld 2002).
En términos generales las concentraciones de $\mathrm{Cd}$ y $\mathrm{Cu}$ (Tabla 1) fueron significativamente influenciadas por la ubicación de la colonia de nidificación y por la edad de las aves (Tabla 2). Sin embargo, a la escala espacial analizada, los factores considerados no tuvieron un efecto significativo sobre la concentración del Cu (Tabla 2). Al no haber diferencias significativas entre los grupos, los datos se agruparon y la gran media de la concentración de $\mathrm{Cu}$ fue calculada en 15,57 \pm 0,079 (rango = 12,10-24,90; $\mathrm{n}=20) \mu \mathrm{g} \mathrm{g}^{-1} \mathrm{ps}$. Estos valores son comparables a los reportados para la gaviota dominicana en estudios del norte de Chile y la Patagonia Argentina. En Chile, se encontraron concentraciones de 10,0-16,6 $\mu \mathrm{g} \mathrm{g}^{-1}$ ps de $\mathrm{Cu}$ en individuos capturados en una zona contaminada por relaves de una empresa minera (Vermeer \& Castilla 1991). En el caso de Argentina, la concentración del metal fue de 9,6-22,3 $\mu \mathrm{g} \mathrm{g}^{-1}$ ps en gaviotas que provenían de una zona silvestre (Gil et al. 2006). Ninguno de los dos estudios citados reportó necrosis hepática. Los valores aquí encontrados y aquellos reportados por Gil et al. (2006) se pueden considerar altos, pero se encuentran en el rango de 14,9-149,0 $\mu_{\mathrm{g} \mathrm{g}}{ }^{-1}$ ps observados en tres especies de vertebrados marinos en la Antártica (SteinhagenSchneider 1986) y en el rango de $10,3-46,8 \mu \mathrm{g} \mathrm{g}^{-1} \mathrm{ps}$ para otras cinco especies de aves del norte de Chile (Vermeer \& Castilla 1991). La ausencia de daños en el hígado, sugiere que los altos niveles de $\mathrm{Cu}$ que se encuentran en la gaviota dominicana no ocasionan alteraciones orgánicas. El Cu es un elemento esencial regulado homeostáticamente por las aves (Sturkies 2000, Burger \& Gochfeld 2002), pero sus efectos subletales a exposiciones prolongadas a diferentes concentraciones son desconocidos.

Tabla 1. Concentración de Cadmio y Cobre $\left(\mu \mathrm{g} \mathrm{g}^{-1} \mathrm{ps}\right)$ en hígados de Larus dominicanus del Sistema Costero de Coquimbo. Los valores corresponden a la media y error estándar (ES) / Cadmiun and copper concentration ( $\mu \mathrm{g} \mathrm{g}^{-1} \mathrm{dw}$ ) in the liver of Larus dominicanus from the Coastal System of Coquimbo, Chile. Values are means and standard error (ES)

\begin{tabular}{llccc}
\hline Colonia & Clase Edad & $\mathrm{n}$ & $\begin{array}{c}\text { Cadmio } \\
\text { Media } \pm \mathrm{ES}\end{array}$ & $\begin{array}{c}\text { Cobre } \\
\text { Media } \pm \mathrm{ES}\end{array}$ \\
\hline Isla Pájaros & Adulto & 5 & $9,84 \pm 0,125$ & $14,83 \pm 0,067$ \\
& Volantón & 5 & $1,54 \pm 0,064$ & $15,89 \pm 0,122$ \\
La Herradura & Adulto & 5 & $9,61 \pm 0,097$ & $16,15 \pm 0,072$ \\
& Volantón & 5 & $<0,01^{*}$ & $15,44 \pm 0,065$ \\
\hline
\end{tabular}

*Los valores en este grupo estuvieron por debajo del nivel de detección del equipo utilizado 
La concentración de $\mathrm{Cd}$ en las gaviotas fue significativamente afectado por la colonia de origen y la edad de los individuos (Tabla 2, interacción $P<0,05$ ), cuyas diferencias se detectaron (prueba de Tukey, $\alpha<$ 0,05 ) entre pollos de ambas colonias, entre pollos y adultos, pero no entre los adultos. En aves marinas el $\mathrm{Cd}$ se considera tóxico cuando se presenta en concentraciones mayores a $5 \mu \mathrm{g} \mathrm{g}^{-1}$ ps (Burger \& Gochfeld 2002). Los niveles de Cd encontrados en los pollos de la Isla Pájaros $\left(1,54 \pm 0,064 \mu \mathrm{g} \mathrm{g}^{-1} \mathrm{ps}\right)$ y Bahía La Herradura $\left(<0,01 \mu \mathrm{g} \mathrm{g}^{-1}\right)$ estuvieron bajo este nivel. Pero en el caso de los adultos la media de la concentración fue de $9,72 \pm 0,110 \mu \mathrm{g} \mathrm{g}^{-1} \mathrm{ps}$ (media agrupada: $\mathrm{n}=10$; rango $0,86-1,17 \mu \mathrm{g} \mathrm{g}^{-1} \mathrm{ps}$ ), que es casi el doble del nivel reportado como tóxico. Sin embargo este valor está en el rango encontrado en la gaviota dominicana del norte de Chile (6,8-36,1 $\mu \mathrm{g} \mathrm{g}^{-1}$ ps, Vermeer \& Castilla 1991) y de la Patagonia argentina (2,7-265,8 $\mu \mathrm{g} \mathrm{g}^{-1} \mathrm{ps}$, Gil et al. 2006). En los ejemplares examinados no se observó daño hepático.

Tabla 2. Resultados de la prueba de MANOVA para las concentraciones de $\mathrm{Cd}$ y $\mathrm{Cu}$ en el hígado de Larus dominicanus, de dos colonias (Isla Pájaros y La Herradura, Coquimbo) y grupos de edades (adultos y volantones) / MANOVA test results for $\mathrm{Cd}$ and $\mathrm{Cu}$ concentrations in the livers of Larus dominicanus from two colonies (Pájaros Island and La Herradura, Coquimbo) and age groups (adults and fledglings)

\begin{tabular}{|c|c|c|c|c|c|}
\hline \multirow[b]{3}{*}{ Colonia (C } & \multicolumn{5}{|c|}{ Multivariado } \\
\hline & Wilks $\lambda$ & \multicolumn{2}{|c|}{$\mathrm{F}$} & GL & \multirow{2}{*}{$\begin{array}{c}P \\
<0,0001\end{array}$} \\
\hline & 0,295 & & 903 & 2,15 & \\
\hline Edad (E) & 0,026 & & 667 & 2,15 & $<0,0001$ \\
\hline \multirow[t]{3}{*}{$\mathrm{C} \times \mathrm{E}$} & 0,336 & & 826 & 2,15 & 0,0002 \\
\hline & \multicolumn{5}{|c|}{ Univariado } \\
\hline & SS & GL & MS & $\mathrm{F}$ & $P$ \\
\hline \multicolumn{6}{|l|}{ Colonia } \\
\hline $\mathrm{Cd}$ & 0,210 & 1 & 0,210 & 33,706 & $<0,0001$ \\
\hline Error & 0,100 & 16 & 0,006 & & \\
\hline $\mathrm{Cu}$ & 0,001 & 1 & 0,001 & 0,107 & 0,748 \\
\hline Error & 0,099 & 16 & 0,006 & & \\
\hline \multicolumn{6}{|l|}{ Edad } \\
\hline $\mathrm{Cd}$ & 3,410 & 1 & 3,410 & 547,534 & $<0,0001$ \\
\hline Error & 0,100 & 16 & 0,006 & & \\
\hline $\mathrm{Cu}$ & 0,001 & 1 & 0,001 & 0,019 & 0,891 \\
\hline Error & 0,099 & 16 & 0,006 & & \\
\hline \multicolumn{6}{|l|}{$\mathrm{C} \times \mathrm{E}$} \\
\hline $\mathrm{Cd}$ & 0,191 & 1 & 0,191 & 30,630 & $<0,0001$ \\
\hline Error & 0,100 & 16 & 0,006 & & \\
\hline $\begin{array}{c}\mathrm{Cu} \\
\text { Error }\end{array}$ & $\begin{array}{l}0,003 \\
0.099\end{array}$ & $\begin{array}{c}1 \\
16\end{array}$ & 0,003 & 0,441 & 0,516 \\
\hline Error & 0,099 & 16 & 0,006 & & \\
\hline
\end{tabular}

La concentración de Cd capaz de producir daños orgánicos y alteraciones en el comportamiento de las aves marinas, es diferente para cada especie y depende de sus hábitos alimenticios y de la posición y complejidad de la trama trófica en la cual participa (Ikemoto et al. 2004, Agusa et al. 2011). Al parecer, las gaviotas son capaces de adecuarse a altas concentraciones de $\mathrm{Cd}$ ya que no se han detectado efectos negativos a nivel individual y poblacional (Muirhead \& Furness 1988, Burger \& Gochfeld 2002). La causa de los altos niveles observados estaría en la ocurrencia de procesos de bioacumulación (Bearhop et al. 2000a).

Las diferencias encontradas en la concentración de Cd entre los pollos de la Isla Pájaros y La Herradura (Tablas 1 y 2) pueden explicarse en función de la dieta de los padres (Bearhop et al. 2000b). Es posible que parte del nivel encontrado en los pollos refleje el grado al que estuvo expuesta la hembra durante la formación del huevo (Sturkies 2000, Muñoz et al. 2003), pero nuestros resultados sugieren un efecto más importante de la alimentación durante el cuidado parental. En esta fase los padres de ambas colonias alimentan a sus pollos principalmente con peces (Ludynia et al. 2005). Sin embargo, las gaviotas de la Isla Pájaros presentan al menos dos fuentes adicionales de alimentación (Ludynia et al. 2005, obs. pers.); depredan sobre huevos y pollos, mayoritariamente de piqueros (Sula variegata) y en menor frecuencia de pingüinos de Humboldt (Spheniscus humboldti), y cleptoparasitan a estas dos especies de aves que se alimentan de peces pelágicos de importancia comercial (Herling et al. 2005, Ludynia et al. 2009). Por lo tanto es posible sugerir que adultos y polluelos de la gaviota dominicana en Isla Pájaros forman parte de una trama trófica compleja, en la cual el Cd se bioacumula y por ello se le encuentra en mayor cantidad en los pollos silvestres.

Los resultados de este estudio son preliminares debido al limitado tamaño de la muestra. Sin embargo, su importancia radica en que aporta información, por primera vez, sobre la concentración de $\mathrm{Cd}$ y Cu en gaviotas de dos grupos de edad y de dos colonias distintas. Los altos niveles de metales encontrados en los adultos de gaviota dominicana, posiblemente se deban a una exposición crónica, cuyo origen (natural o antropogénico) debiera ser analizado al igual que sus efectos subletales y subclínicos. Por lo tanto, se recomienda el establecimiento de planes de monitoreo de metales pesados en el SCC, considerando el uso de adultos como bioindicadores a una mesoescala regional, y a los pollos de distintas colonias como indicadores de las concentraciones a una escala espacial pequeña. 


\section{Agradecimientos}

A Fabrizio Queirolo y Susana Stegen por facilitar su laboratorio y asesoría en los análisis químicos. A Felipe Sepúlveda por su activa y generosa colaboración en el trabajo de campo. Este estudio fue financiado por el proyecto FONDECYT 1010250 de GL-J.

\section{LITERATURA CITADA}

Agusa T, K Nomura, T Kunito, Y Anan, H Iwata \& S Tanabe. 2011. Accumulation of trace elements in harp seals (Phoca groenlandica) from Pangnirtung in the Baffin Island, Canada. Marine Pollution Bulletin 63: 489-499.

Bearhop S, S Waldron, DR Thompson \& WR Furness. 2000a. Bioamplification of mercury in great skua Catharacta skua chicks: the influence of trophic status as determined by stable isotope signatures of blood and feathers. Marine Pollution Bulletin 40: 181-185.

Bearhop S, RA Phillips, DR Thompson, S Waldron \& WR Furness. 2000b. Variability in mercury concentrations of great skuas Catharacta skua: the influence of colony, diet and trophic status inferred from stable isotope signatures. Marine Ecology Progress Series 195: 261-268.

BirdLife. 2011. Species factsheet: Larus dominicanus. BirdLife International. [en línea] <http://www.birdlife.org on 17/01/ 2011>

Bravo M, M Gallardo, G Luna-Jorquera, P Núñez, N Vásquez \& M Thiel. 2009. Anthropogenic debris on beaches in the SE Pacific (Chile): Results from a national survey supported by volunteers. Marine Pollution Bulletin 58: 1718-1726.

Burger J \& M Gochfeld. 2002. Effects of chemicals and pollution on seabirds. In: Schreiber EA \& J Burger (eds). Biology of marine birds, pp. 485-525. CRC Press, Boca Raton.

Gil M, A Torres, M Harvey \& L Esteves. 2006. Metales pesados en organismos marinos de la zona costera de la Patagonia argentina continental. Revista de Biología Marina y Oceanografía 41: 167-176.

Harrison P. 1983. Seabirds, and identification guide, 448 pp. Houghton Mifflin Company, Boston.

Herling C, B Culik \& J Hennicke. 2005. Diet of the Humboldt penguin (Spheniscus humboldti) in northern and southern Chile. Marine Biology 147: 13-25.

ICES. 1999. Seabirds as monitors of marine pollution. En: ICES (ed). Report of the Working Group on Seabird Ecology, ICES Headquarters, 22-26 March 1999. ICES CM 1999/C:5: 2353.

Ikemoto T, T Kunito, H Tanaka, N Baba, N Miyazaki \& S Tanabe. 2004. Detoxification mechanism of heavy metals in marine mammals and seabirds: Interaction of selenium with mercury, silver, copper, zinc, and cadmium in liver. Archives Environmental Contamination and Toxicology 47: 402-413.
Kozertka J. 2002. Räumliche und zeitliche Aktivitätsmuster der Dominikanermöwe (Larus dominicanus) in Nordchile, 81 pp. Diplomarbeit Institut für Meereskunde, Kiel.

Ludynia K, S Garthe \& G Luna-Jorquera. 2005. Seasonal and regional variation in the diet of the Kelp Gull in Northern Chile. Waterbirds 28: 359-365.

Ludynia K, S Garthe \& G Luna-Jorquera. 2009. Distribution and foraging behaviour of the Peruvian Booby (Sula variegata) off northern Chile. Journal of Ornithology 151: 103-111.

Mahoney S \& J Jehl. 1984. Body water content in marine birds. The Condor 86: 208-209.

Moriarty F. 1983. Ecotoxicology. The study of pollutants in ecosystems, 333 pp. Academic Press, London.

Muirhead SJ \& RW Furness. 1988. Heavy metal concentrations in the tissues of seabirds from Gough Island, South Atlantic Ocean. Marine Pollution Bulletin 19: 278-283.

Muñoz J \& PH Becker. 1999. The Kelp Gull as bioindicator of environmental chemicals in the Magellan region . A comparison with other coastal sites in Chile. Scientia Marina 63: 495-502.

Muñoz J, PH Becker, U Sommer, P Pacheco \& RP Schlatter. 2003. Seabird eggs as bioindicators of chemical contamination in Chile. Environmental Pollution 126: 123-137.

Ochoa-Acuña H, MS Sepúlveda \& TS Gross. 2002. Mercury in feathers from Chilean birds: influence of location, feeding strategy, and taxonomic affiliation. Marine Pollution Bulletin 44: 340-349.

Quinn GP \& MJ Keough. 2002. Experimental design and data analysis for biologists, 537 pp. Cambridge University Press, Cambridge.

Scheiner S. 1993. MANOVA: Multiple response variables and multispecies interactions. En: Scheiner SM \& J Gurevitch (eds). Design and analysis of ecological experiments, pp. 94-112. Chapman \& Hall, New York.

Shealer DA. 2002. Foraging behavior and food of seabirds. En: Schreiber EA \& J Burger (eds). Biology of marine birds, pp. 137-177. CRC Press, Boca Raton.

Simeone A, G Luna-Jorquera, M Bernal, S Garthe, F Sepúlveda, R Villablanca, U Ellenberg, M Contreras, J Muñoz \& T Ponce. 2003. Breeding distribution and abundance of seabirds on islands off north-central Chile. Revista Chilena de Historia Natural 76: 323-333.

Stegen S, F Queirolo, S Cortés, J Pastenes, P Ostapczuk, F Backhaus \& C Mohl. 2000. Use of the fresh water plants Zannichellia palustris and Myriophyllum acuaticum for biomonitoring of $\mathrm{Cd}, \mathrm{Pb}$ and $\mathrm{Cu}$ in Anden Rivers of Chile. Boletín de la Sociedad de Chilena de Química 3: 449-459.

Steinhagen-Schneider G. 1986. Cadmiun and copper levels in seals, penguins and skuas from the Weeddel Sea in 1982/ 1983. Polar Biology 5: 139-143. 
Sturkies P. 2000. Avian physiology, 685 pp. Causey Whittow, New York.

Thiel M, M Bravo, IA Hinojosa, G Luna-Jorquera, L Miranda, P Núñez, AS Pacheco \& N Vásquez. 2011. Anthropogenic litter in the SE Pacific: an overview of the problem and possible solutions. Journal of Integrated Coastal Zone Management 11: 115-134.

Vermeer K \& JC Castilla. 1991. High cadmium residues observed during a pilot study in shorebirds and their prey downstream from the EI Salvador copper mine, Chile. Bulletin of Environmental Contamination and Toxicology 46: 242248.
Villablanca R, G Luna-Jorquera, VH Marin, S Garthe \& A Simeone. 2007. How does a generalist seabird species use its marine habitat? The case of the kelp gull in a coastal upwelling area of the Humboldt Current. ICES Journal of Marine Science 64: 1348-1355.

Weichler T, S Garthe, G Luna-Jorquera \& J Moraga. 2004. Seabird distribution on the Humboldt Current in northern Chile in relation to hydrography, productivity, and fisheries. ICES Journal of Marine Science 61: 148-154.

Zúñiga M. 1998. Ecotoxicología de metales en el ambiente acuático: Una revisión actualizada. En: Arcos D (ed). Minería del cobre, ecología y ambiente costero, pp. 349-376. Editora Aníbal Pinto, Santiago.

Recibido el 21 de enero de 2011 y aceptado el 24 de junio de 2011 